\title{
BACKBONE MAPPING RESOURCES, SERVICES AND APPLICATIONS FOR INFORMATION SUPPORT OF REGIONAL MANAGEMENT
}

\author{
Dr. Oleg Yakubailik ${ }^{1,2}$ \\ ${ }^{1}$ Institute of Computational Modelling of the Siberian Branch of the Russian Academy of Sci- \\ ences (ICM SB RAS), Russia \\ ${ }^{2}$ Siberian Federal University, Russia
}

\begin{abstract}
The work considers information and computing technologies supporting regional decisions making and based on geoinformation web-systems and mapping web-services. The use of such systems for the information support of regional management is now becoming common. Long-term strategic forecasting and planning of territories development, solution of various institutional and sectoral problems today are often based on the use of integrated information and computing environment, complex information systems of regional management, which are based on geospatial (mapping) data.

With all the variety of tasks to be solved, methods and approaches used, information technologies and software products, it is possible to distinguish several characteristic features of IT solutions used in the field under consideration. Such regional geoinformation web-systems provide access to huge arrays of geospatial information and services distributed in the Internet, remote data processing with high performance multi-user computers. Usually they are built in service-oriented architecture, and they can be regarded as a set of interrelated software components for spatial data operations, such as import/export, cataloging, visualizing, creating, processing, distribution, etc. Different geospatial platform may be used as a basis - from commercial software (for example - ESRI ArcGIS and its components) to free and open source geospatial software stack, such as OSGeo projects.
\end{abstract}

This paper discusses mapping resources, technologies and web-services developed for regional authorities of Kranoyarsk Territory, Russia. In particular, over the past few years, about a dozen regional-level geoinformation web-systems have been created, including "Geoinformation system for monitoring the state of the environment in the zone of operation of the oil and gas industry enterprises of the Krasnoyarsk Territory", "The network of regional educational services", "The health map of the Krasnoyarsk Territory", "Satellite Remote Sensing data archive of the Krasnoyarsk Territory", "Automated system for monitoring the municipalities in economy and regional development".

The modular architecture of the systems under consideration, the use of standard web services for data exchange between these modules, ensures the rapid adaptation of the existing software to the customer requirements, the replication of individual components, and their complementarity. In its turn, registration of created resources, i.e. spatial data in the centralized geoportal catalog, provides the possibility of their simultaneous application in several developments. This service-oriented approach, based on the active implementation of web technologies into application information systems, is increasingly being used now. 


\section{INTRODUCTION}

Modern web mapping software (spatial web system, web GIS) are usually built according to so-called service-oriented architecture, and they can be considered as a set of interrelated software components for operations with spatial data, such as import/export, cataloging, visualizing, creating, processing, distribution, etc. The technological basis for these solutions is a set spatial data processing and visualization software such as Google Maps API, Mapserver Mapscript, Yandex Maps API, Scanex GeoMixer, etc. These programming components form the web mapping user interface and provide access to the different functions of mapping elements on web page, such as customization of map visualization, selection of thematic data from predefined data sets, changing the data of the presented map features, and so on. [1]. Web mapping applications have become very popular due to high degree of the user interface interactivity, very fast regeneration of picture on the screen during moving the map an changing its scale, different other operations with spatial data. It is also important the support of spatial data exchange formats and standards, such as KML, WMS, GeoJSON, etc. [2].

Web services have become the key element of these systems. The most popular at the moment concept of building mapping web applications involves the creation of an interconnected web services components set, the implementation of which is carried out simultaneously on the server and the client computer (multi-tier architecture), as well as formation of a spatial data set usually made in the format of popular GIS or using a specialized geospatial database. The initial spatial data preparation for the web application is usually carried out outside the considered web-system; for this, standard desktop GIS are typically used today (ArcGIS, QGIS, MapInfo and others) [3].

\section{REGIONAL SPATIAL DATA INFRASTRUCTURE}

Spatial data and web-based systems are widely used in regional management tasks at present. The range of interests is very wide-agriculture, environmental problems, territory planning, transport management, emergencies, etc. [4-9].

Research and development in the field of the spatial data infrastructure on the regional level have been carried out at Institute of Computational Modeling of Siberian Branch of Russian Academy of Sciences (ICM SB RAS) at Krasnoyarsk (Russia) for somewhere 20 years now. This work resulted in the creation of software and technological tools for applied geoinformation web-system (geoportals) construction. The procedure of quick implementation of applied geoinformation web-systems has been developed. The appropriate technological, informational and computational software, such as storage, processing and spatial data (including satellite images) analyzing services, along with applied program and user interface repositories, access authorization media within the structure of the geoportal and spatial data catalogue, cartographic web-visualization system, auxiliary and applied web-services have been created [10].

Software and technological tools that are being developed represent high-level repositories of functions and classes, application templates directed at the end user. These provide an operational solution of the following tasks:

- Maintenance and storage of digital cartographic materials, raster images of the location enabling the univocal addressing and positioning of objects in the regional (municipal) infrastructure; 
- Navigation through informational cartographic resources, visualization and analysis of spatially oriented data on unified digital maps;

- Interaction with cartographic and attributive resources (databases) of third party applied information systems;

- Solution to various spatial tasks using resources of the spatial data storage (spatial search, object creation etc.);

- Provision of access to the system using modern geoinformation system technologies and interfaces.

Using the experience of successful completed projects, let's try to define main features of web mapping systems focused on regional management tasks from the functional point of view -

- The system should consist of client and server parts, thereby realizing the "clientserver" technology. Application of the MVC (Model-View-Controller) design pattern as the server part basis provides ample opportunities for the tasks solution. Using of this architecture assumes separation of the application data, user interface and control logic into three separate components. Modification of each component can be performed independently. Apart from the standard MVC elements, key units of the system are also page presentation templates and AJAX handlers.

- Spatial data should be recorded in the resource catalogue. The web application communication with the resource catalogue is performed on the basis of service-oriented architecture using the SOAP/XML protocol. It creates a set of functions available in the form of application programming interface (API), which offers the possibility of resources searching, filtering, management, editing, copying, moving, etc.

- Two API sets should be formed. The 1-st API is common (client) interface for user applications, and the 2-nd one is for advanced (server) interface for applications having the ability to control the resources catalogue, its objects and relationships between objects.

Information support for the applied regional geoinformation web systems consists of several types of geospatial and other data and appropriate tools of their processing [11]:

- tabular data stored in the database - it provides the business logic of the system, its basic content;

- thematic spatial data related to direct content of the application system objectives and functions. This type of data can be created both within the applied system user interface (based on the web browser or Windows application) and by tools of third-party applications - various GIS/CAD programs, etc.

- mapping background - information resources of auxiliary character maps and satellite images used for the thematic spatial data visualizing. Own data sets can be used as well as third-party services provided by Yandex, Google, 2GIS and other companies.

- tools for creating and managing the spatial metadata and related services of visualization, search and filtering.

- procedures for geospatial data classifying - standardized classification system enables multiple data using for various projects.

- tools for the geospatial data web publication managing - user interfaces for the formation of geoportal thematic sections based on the web-content management system. 
- tools for the systems integration, information exchange and interaction based on the geoportal information-analytical and mapping services.

Particular attention should be paid to spatial metadata. Metadata is data about data, i.e. catalogues, directories, registers, metadata-bases and other forms of descriptions of digital and analogue data sets comprising information on its composition, content, status (actuality and updatableness), on its genesis (ways and conditions of its origination), on its location, quality (its completeness, consistency and validity), on its formats and forms of presentation, on access, on acquisition and usage conditions, on copyright, property and related rights for this data. Metadata databases, including those comprised in cartographic data and geoinformational systems, can provide a medium of inventory of informational resources, be involved in existing informational systems and databases, as well as they can be used in searching and estimating the origins of spatial data.

To solve regional management tasks under consideration, the formal definition of the following major components of the spatial data infrastructure should be performed [12]:

- Basic spatial data that can provide the base for location (positioning) of all other spatial and non-spatial (attributive or thematic) ones;

- Spatial metadata and accessing mechanisms that include metadata databases located in the network of servers, gateways and portals, available through the information interchange centres and facilitating data search among the multitude of storages, funds, databases located at their bearers, as well as they include standards and programming tools for metadata-base creation and accessing;

- Standards on spatial data, including standards on spatial data models, geodetic provision, spatial and temporal coordinate systems, representation formats in spatial databases, interchange formats, metadata, data accuracy and quality, digital maps and cartographic methods, classifiers of particular thematic data types up to standards on notion and nomenclature instrument.

\section{DESIGNED SOFTWARE STRUCTURE}

The geoportal software is the key element of the presented software and technological tools for applied geoinformation web-system development. It includes a number of components that are currently running on different nodes in distributed architecture [13] -

- Data Storage Subsystem within which the support of all the formats of popular GIS (ArcGIS SHP, MapInfo TAB, etc.) and spatial databases, such as PostgreSQL / PostGIS and others is provided.

- Geoportal Resources Catalogue, which is a metadata management subsystem, a database and a program repository set (API) for various operations with resources on the base of web-services on SOAP protocol.

- Geoportal Data Management Subsystem. The main task is the registration of information resources in the Catalogue, entering and editing of metadata. Further functions relate to the distinctions of access rights, metadata import from third party WMSresources and its applicable registration in the catalogue and others.

- Geoportal Metadata User Web-interface, which is a web-application designed for navigation through the resources registered in the system, and search among these resources. It provides navigation though the Resource Catalogue with regard to the 
multiple resource classification and filtration on different criteria, as well as creation of user data sets ("Shopping Cart") and others.

- Cartographic Web-interface Subsystem, which is a web-application for representation of maps, spatial data layers though the web-interface (Fig. 1). A capability to mark various background cartographic base maps such as Yandex, Google, ESRI, 2GIS and others (more than 40 items) should be specially noted.

- Web-publication Subsystem. It's the set of tools for preparation of map data for web presentation outside the geoportal. This part of software is based on Drupal web content management system.

- Cartographic web-services, which include advanced address search with regional specifics, geocoding, routing, watercourse constructing and others. These web-based services are designed for work carried out as a part of third party applied geoinformation systems.

Presented geoportal software has several types of public (user and program) interfaces:

- User web interface for a standard web browser;

- Cartographic web services based on the OGC international standards;

- Application Program Interfaces (API) for geoportal cartographic data visualization;

- Informational services for third party applied systems.

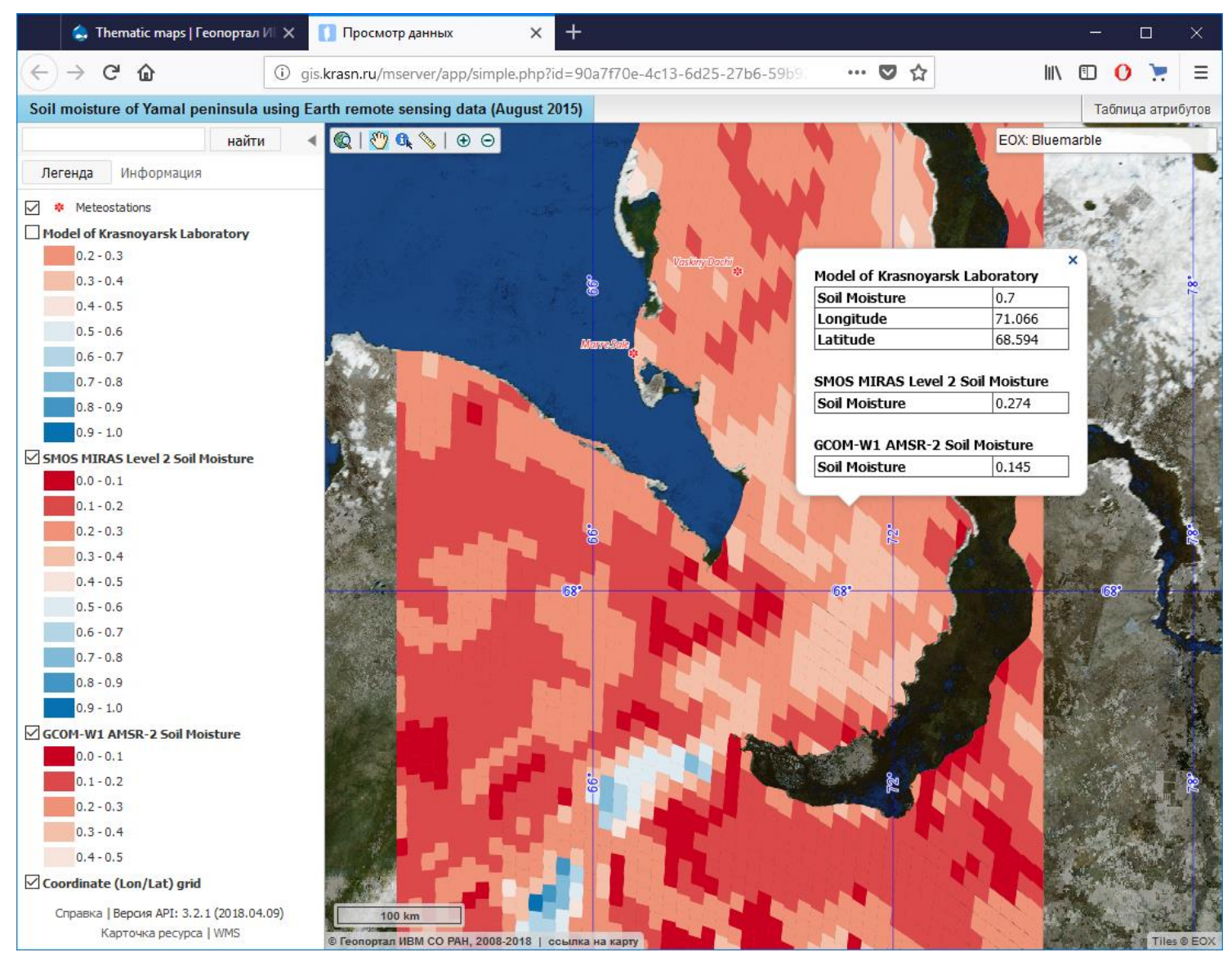

Figure 1. Web mapping user interface of the Geoportal of ICM SB RAS. 
For the direct access to spatial data the protocols Open Geospatial Consortium (OGC), such as Web Map Service (WMS), Web Map Tiling Service (WMTS) and others are used; they allow access to the geoportal resources from applied user programs directly such as ArcGIS, MapInfo, QGIS etc., as the support of these protocols is integrated into most of the modern geoinformation systems.

Thus, at first the users can become acquainted with thematic maps via the interface, and then link them by a web-service to their geoinformation system project for data analyzing: geoinformation software, such as an application for Windows, is usually used for this purpose. OGC protocols imply not a "picture" transition via a web-service only, but also running queries on table object data of the linked map layers.

\section{IMPLEMENTATION EXPERIENCE}

The developed system of applied high-level services successfully established itself as a software technological basis of information analytical systems for regional territory and municipal authorities. A number of such systems were implemented, such as decision support systems for local government on health, education, transport and disaster management; environmental monitoring and assessment of the state of the environment; support system for socio-economic development of the region. In general, during the last years it was implemented more than 10 analytical applications. In particular, over the past few years, about a dozen regional-level geoinformation web-systems have been created, including "Geoinformation system for monitoring the state of the environment in the zone of operation of the oil and gas industry enterprises of the Krasnoyarsk Territory", "The network of regional educational services", "The health map of the Krasnoyarsk Territory", "Satellite Remote Sensing data archive of the Krasnoyarsk Territory", "Automated system for monitoring the municipalities in economy and regional development". Customers of these systems are the departments of the government of the Krasnoyarsk region, one of federal subjects of Russia. A few more development was established within the framework of the scientific research grants.

Bodies of executive authorities are customers of these systems. Implementation of systems is carried out taking into account features and specifics of customers. Typically, the installation software runs on the customer's equipment, but it is also possible to use own data center of developers. Such placement is in some cases more appropriate given, for example, the nearby situated satellite imagery archives, which are very large.

The web-GIS can provide access to the spatial analysis for users who are not technical experts in GIS. It is very attractive to see your data in a spatial representation without using any complicated programs and being limited only by a standard web browser. For example, the initial data for the Krasnoyarsk region fire situation-mapping interface is constantly real time updated departmental database on fires in the region. To ensure the operative data presentation in the geoinformation system, a special web-service providing the necessary pre-processing and raw data aggregation was developed (Fig. 2). There are a number of similar system around the world. 


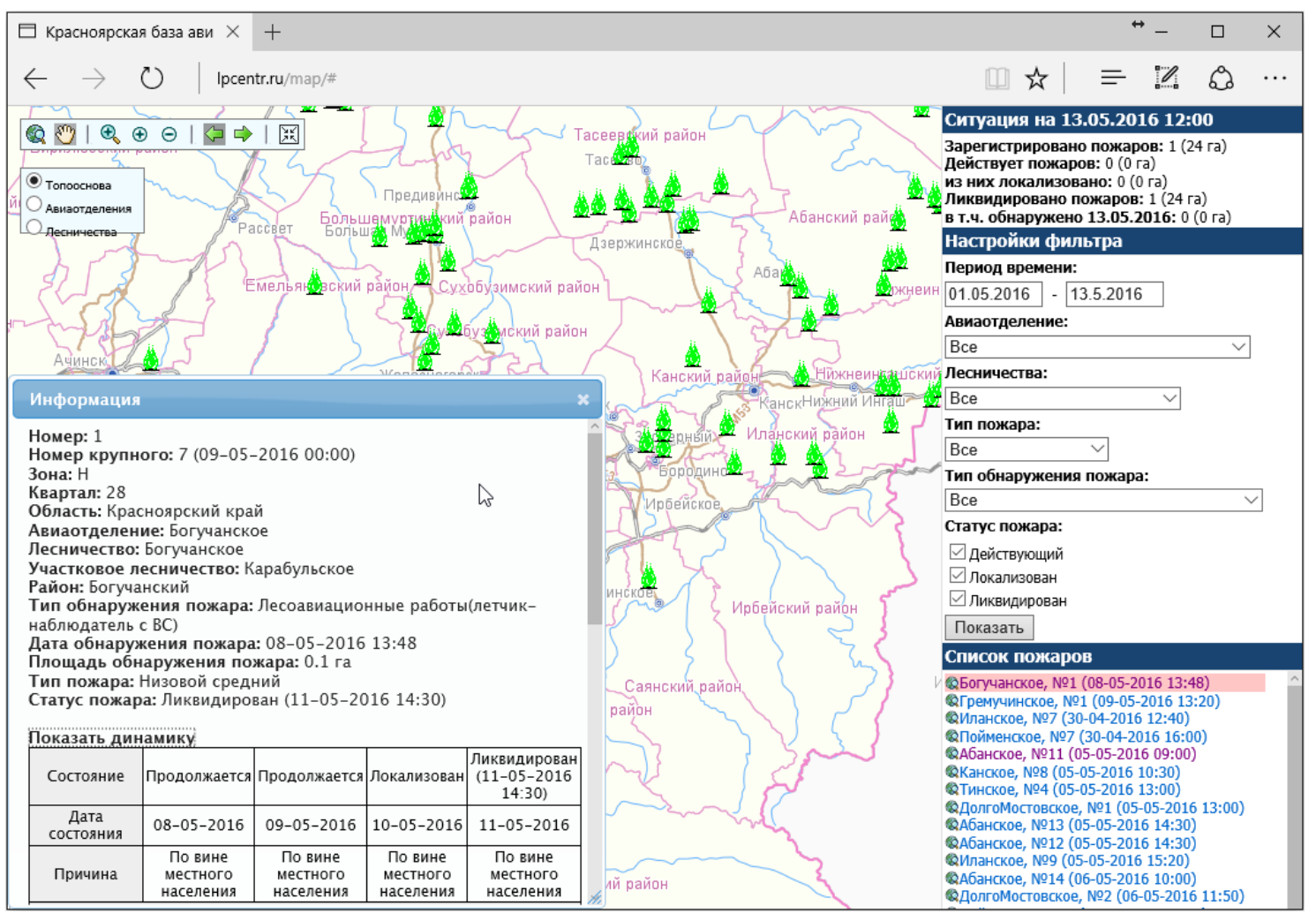

Figure 2. Krasnoyarsk region operative fire situation web mapping interface.

\section{CONCLUSION}

The developed software and technological solutions have successfully proven themselves as a basis for resource intensive information and analytical systems of regional level for various problems solution, such as sectoral management information support (in the sphere of health, education, transport), environmental monitoring and the environment state assessment, forecasting of the region's socio-economic development, centralized map data information supplying.

The modular architecture of these systems, the use of standard web-services to exchange the data between these modules provide rapid adaptation of the existing software to customer's requirements, replication of individual components, their complementarity. In turn, the registration of created resources - spatial data - in the geoportal-centralized catalog provides the possibility of its simultaneous use in multiple developments. This service-oriented approach based on active web technologies implementation in the applied information systems is increasingly used today.

\section{REFERENCES}

[1]. Pinde Fu. Web GIS: principles and applications / Pinde Fu, Jiulin Sun // Esri Press, 380 New York Street, Redlands, California 92373-8100.

[2] Songnian Li. Advances in Web-based GIS, Mapping Services and Applications / Li Songnian, Suzana Dragicevic, Bert Veenendaal // CRC Press, 2011. 
[3] Sample J.T. Geospatial Services and Applications for the Internet / J.T. Sample, K. Shaw, S. Tu, et al// Springer-Verlag US, 2008. - 179 p.

[4] Huysegoms L, Cappuyns V. Critical review of decision support tools for sustainability assessment of site remediation options // Journal of Environmental Management, V. 196, 2017, pp. 278-296.

[5] Grêt-Regamey A., Sirén E., Brunner S.H., Weibel B. Review of decision support tools to operationalize the ecosystem services concept // Ecosystem Services, V. 26, Part B, 2017, pp. 306-315.

[6] Silva S., Alçada-Almeida L., Dias L.C. Development of a Web-based Multi-criteria Spatial Decision Support System for the assessment of environmental sustainability of dairy farms // Computers and Electronics in Agriculture, V. 108, 2014, pp. 46-57.

[7] Mari R., Bottai L, Busillo C, Calastrini F. et al. A GIS-based interactive web decision support system for planning wind farms in Tuscany (Italy) // Renewable Energy, V. 36, Issue 2, 2011, pp. 754-763.

[8] R. Kingston, S. Carver, A. Evans, I. Turton. Web-based public participation geographical information systems: an aid to local environmental decision-making // Computers, Environment and Urban Systems, V. 24, Is. 2, 2000, pp. 109-125.

[9] Rinner C., Keßler C., Andrulis S. The use of Web 2.0 concepts to support deliberation in spatial decision-making // Computers, Environment and Urban Systems, V. 32, Is. 5, 2008, pp. 386-395.

[10] Yakubailik O.E., A. Kadochnikov A.A., Tokarev A.V. Applied software tools and services for rapid web GIS development // International Multidisciplinary Scientific GeoConference (SGEM 2015). SGEM2015 Conference Proceedings - 2015. - Book 2. Vol. 1. - P. 487-494.

[11] Yakubailik O. Geospatial services \& Web GIS software for environmental monitoring problems // International Multidisciplinary Scientific GeoConference (SGEM 2016). SGEM2016 Conference Proceedings - 2016. - Vol. 1. № 2 - P. 657-664.

[12] Yakubailik O.E. The Formation of Regional Spatial Data Infrastructure // Journal of Siberian Federal University. Engineering \& Technologies, 2016, V. 9, № 7, pp. 979-986.

[13] Yakubailik O.E., A. Kadochnikov A.A., Tokarev A.V. Web Technologies for the Geoinformation System of Rapid Assessment of Atmospheric Pollution and Climatic Conditions in Krasnoyarsk // CEUR Workshop Proceedings 2017, Vol. 2033, pp. 44-48. 